\title{
Resonances and multi-particle states
}

\section{Michael Döring*}

Helmholtz-Institut für Strahlen- und Kernphysik (Theorie) and

Bethe Center for Theoretical Physics, Universität Bonn, D-53115 Bonn, Germany

E-mail: doering@hiskp.uni-bonn.de

The spectrum of excited hadrons starts to become accessible on the lattice. Resonance phase shifts can be extracted with Lüscher's method and extensions thereof that allow to address multichannel scattering problems. To determine the amplitude, methods like moving frames or modified boundary conditions allow for more eigenvalues in the finite volume, but often at different energies. Interpolations in energy relate different eigenvalues and help to pin down the amplitude. Expanding the $T$ - or the $K$-matrix locally provides a controlled scheme by removing the known non-analyticities of thresholds. This can be stabilized by using Chiral Perturbation Theory. Different examples to determine resonance pole parameters and to disentangles resonances from thresholds are discussed, like the scalar mesons $f_{0}(980), \sigma(600)$, and excited baryons $N(1535) 1 / 2^{-}$ and $\Lambda(1405)$.

31st International Symposium on Lattice Field Theory LATTICE 2013

July 29 - August 3, 2013

Mainz, Germany

${ }^{*}$ Speaker. 


\section{Introduction}

One of the present issues in QCD lattice simulations is to determine the spectrum of excited hadrons. Increased computational power allows to extract not only the ground state levels but also a tower of excited states, in combination with techniques like multi-hadron operators in generalized eigenvalue problems. As quark masses drop, excited levels are often in kinematic regions where two or more particles can be simultaneously on-shell, i.e. where resonances acquire a width and can decay to one or more channels.

Many efforts to determine the spectrum of excited hadrons have been carried out in the last years [1-32]. In the volume-dependent spectrum of eigenvalues, "avoided level crossing" is usually taken as a signal of a resonance, but this criterion has been shown insufficient for resonances with larger widths [33-37]. For resonances with a single, two-body decay channel, one often uses Lüscher's approach to extract phase shifts from the discrete energy levels in the box [38, 39]. This method allows to extract the phase at the energy of the eigenvalue, but phases at different energies are needed to determine the precise resonance properties, in particular the width. If the resonance is sufficiently far away from thresholds and in the absence of background, a simple BreitWigner parameterization allows to describe the phase shift and to determine the width. The prime examples are given by the $\rho(770)$ [40-44] and also the $K^{*}(892)[45,46]$. In such cases, the phase passing through 90 degree can be taken as criterion for the approximate resonance mass. However, apart from resonances like the $\rho(770), K^{*}(892)$ or $\Delta(1232)$, the background is in in general not negligible and energy-dependent itself, and the connection between the phase transition through $\pi / 2$ and the resonance mass is lost. The resonance shape can also be heavily distorted from the Breit-Wigner case by large branching ratios into close-by inelastic channels like in case of the $N(1535) 1 / 2^{-}$with its strong coupling to the $\eta N$ state. Here, an additional complication arises from the close-by $N(1650) 1 / 2^{-}$that interferes with the $N(1535) 1 / 2^{-}$through the open decay channels that both resonances share. Another example is the Roper resonance $N(1440) 1 / 2^{+}$that decays considerably into the multi-particle $\pi \pi N$ state. In general, the well-defined resonance properties are the pole position in the complex plane of the scattering energy. The real part of the position can be regarded as the resonance mass, while the imaginary part is a measure for the width. The residues associated with the resonance pole factorize channel-wise and can therefore be related to the branching ratios.

If more than one two-body decay is possible, the reconstruction of the resonance, or in general the multi-channel scattering amplitude, becomes more complicated. For example, in isoscalar $S$ wave meson-meson-scattering, there are at least two relevant channels, $\pi \pi$ and $K \bar{K}$. Obviously, close to and above the $K \bar{K}$ threshold, one has three independent transitions $\pi \pi \rightarrow \pi \pi, K \bar{K} \rightarrow$ $K \bar{K}$ and $\pi \pi \rightarrow K \bar{K}, K \bar{K} \rightarrow \pi \pi$ with the off-diagonal transitions being equal due to time-reversal invariance. To determine the amplitude, one needs, thus, three measurements at the same energy, instead of one as in the one-channel case. It is little practical or even impossible to tune different lattices such that three independent measurements at a given energy can be taken. Interpolations in energy can provide the needed information, with minimal assumptions on the smoothness of the $K$-matrix and potentially in combination with Chiral Perturbation Theory or twisted boundary conditions [36, 47-49]. This will be discussed in these proceedings.

A classical case, where the multi-channel treatment is mandatory to understand the dynamics, 
is given in the $S$-wave, scalar-isoscalar sector with the $f_{0}(980)$ state just at the $K \bar{K}$ threshold. Similarly, the isospin-one $S$-wave $a_{0}(980)$ resonance is close to the $K \bar{K}$ threshold. To disentangle these states from thresholds is demanding as discussed in Sec. 2, particularly because $S$-wave thresholds manifest themselves in the same avoided level crossing as narrow resonances.

Examples for the relevance of multi-channel dynamics in the baryon sector are the $N(1535) 1 / 2^{-}$ and the $\Lambda(1405)$. The $N^{*}$ resonance has an experimentally known large branching ratio into the $\eta N$ channel (threshold energy: $1486 \mathrm{MeV}$ ). Furthermore, the $K \Lambda$ and $K \Sigma$ channel play an important role in this partial wave. The channel dynamics becomes particularly intricate when extrapolating to unphysical pion masses, because the threshold energies depend differently on the quark masses. The consequences are discussed in Sec. 3.

The $\Lambda(1405)$ is an $S$-wave resonance with strong couplings to the $\bar{K} N$ and the $\pi \Sigma$ channel. Its precise properties continue to be under debate, as it is difficult to directly access experimentally (only through measurements of $\pi \Sigma$ invariant masses). The strong coupled-channel dynamics might even lead to a much-discussed two-pole structure of this resonance [50]. The structure of the resonance in the finite volume and possibilities to extract its properties are discussed in Sec. 3. For recent results on the $\Delta(1232)$ decay width, see Ref. [51].

As discussed, the determination of the multi-channel amplitude requires multiple measurements on the lattice, in combination with additional techniques such as interpolation in energy. A related issue is the mixing of partial waves due to the breaking of rotational symmetry on the lattice. A given eigenvalue is not tied to one partial wave but to a tower of partial waves. Those can be classified by the groups of cubic symmetry. The problem becomes more complicated once moving frames are considered. This techniques allows to determine more eigenvalues from the same lattice, but partial wave mixing is more severe than in the unboosted case. For the first few boosts, one still has subgroups of cubic symmetry, the so-called little groups. As the artifacts tied to moving frames can be precisely reconstructed, the method has proven to be a powerful tool in lattice QCD (in practice, always combined with a truncation of higher partial waves). However, to disentangle, e.g., the $S$-wave from the $P$-wave, one has to have two measurements at the same energy: the $P$ wave can be determined independently from the $S$-wave. With the knowledge of the $P$-wave, the measurement of the mixed $S P$-wave eigenvalue allows to deduce the $S$-wave amplitude.

Thus, like in the multi-channel case, multiple measurements on the lattice are required, again in combination with energy-interpolation techniques, potentially stabilized by the use of Chiral Perturbation Theory. Implications and practical examples are discussed in Sec. 2.2.

Apart from coupled channels of two-body intermediate states, there can be three or more particles simultaneously on-shell. For example, the four-pion threshold is even below the $K \bar{K}$ threshold, though it is known that its contribution is small and often neglected. In the sector of excited baryons, $\pi \pi N$ dynamics is known to play a crucial role for the Roper resonance $N(1440) 1 / 2^{+}$. Here, the two pions and the nucleon can all be in relative $S$-waves. The absence of the centrifugal barriers leads then to a very early onset of inelasticities into the $\pi \pi N$ channel, as it is known from partial wave analyses [52]. Finite-volume effects are expected to play a major role and might lead to drastic changes of the eigenvalue spectrum once extrapolating to the physical point. This is because the resonance and the three-body threshold are close to each other. Indeed, it is known for a long time that the non-trivial shape of the Roper resonance is closely tied to three-body dynamics, or, in other words, to branch points in the complex plane [53] close to the resonance pole position. 
Multi-particle states on the lattice are discussed in Sec. 4.

In the following, implications of coupled-channel scattering and multi-particle states for the finite volume are discussed and connections to the works presented at the Lattice 2013 conference are made.

\section{Excited mesons: multi-channel scattering}

In data analysis it is advantageous to separate the known non-analytic threshold openings from the full amplitude $T$. This can be achieved by schematically writing

$$
T=V+V G T
$$

where the kernel $V$ and the two-body propagator $G$ are matrices in channel space. The kernel $V$ is a smooth function at the (in)elastic thresholds and $G$ contains non-analyticities. If $G$ is only given by the phase space factor (purely imaginary above threshold and real below), one has a $K$-matrix approach. Dispersive real contributions to $G$ can be included by writing a dispersion relation, but also then the amplitude can be recast in the $K$-matrix formulation [36]. The imaginary part of $G$ ensures also coupled-channel two-body unitarity. In any case, the fact that $V$ is a smooth function permits to develop it locally in a power series of the energy. In the Chiral Unitary Approach the series is matched to CHPT order-by-order. When it comes to the analysis of data, this helps to stabilize the extraction of the amplitude.

In the finite volume, momenta are restricted to discrete values due to the periodic boundary conditions of the box. This modifies both $V$ and $G$, but some changes are suppressed exponentially with the box size $L, e^{-M_{\pi} L}$. These (model-dependent) contributions can be separated from the dominant contributions, resulting in a model-independent connection between eigenvalues and phase shifts as derived by Lüscher [38, 39]. The connection between the notation implied in Eq. (2.1) and the Lüscher formalism can be found, e.g., in Ref. [37]. The integration over momenta in the twobody propagator $G$ is reduced to a sum due to the periodic boundary conditions in the finite volume, $G \rightarrow \tilde{G}$. The kernel $\tilde{V}$ contains only exponentially suppressed contributions to finite-volume corrections that can be neglected for sufficiently large box sizes [54], $\tilde{V}=V$. Then, the eigenvalues in the finite volume are given by the poles of $\tilde{T}$ defined by $\tilde{T}=V+V \tilde{G} \tilde{T}$, from which $V$ can be deduced and inserted in Eq. (2.1) to provide the $T$-matrix in the infinite volume. Phase shifts and inelasticities follow.

A practical example of how the expansion of the kernel $V$ can be used to determine the amplitude is given in Ref. [37] for the $f_{0}(980)$. This case is particularly interesting because the resonance is situated closely to the $S$-wave $K \bar{K}$ threshold. The latter manifests itself in the same avoided level crossing as a narrow resonance. Indeed, in Ref. [37] it has been shown that by removing the $f_{0}(980)$ the amplitude changes drastically but the level spectrum in the finite volume shows only rather small changes and the qualitative $L$-dependence of the levels stays the same. In Refs. [36, 37] it has been proposed to modify the boundary conditions for the strange quark to remove the influence of the threshold. In the notation used here, this results in a modified $\tilde{G}$. In particular, instead of allowed momenta $\vec{q}=\frac{2 \pi}{L} \vec{n}, \quad \vec{n} \in \mathbb{Z}^{3}$ for periodic boundary conditions, one has the momenta $\vec{q} \rightarrow \vec{q}+\vec{\theta} / L$ where $\vec{\theta}$ is the twisting angle that can be chosen to vary the $\bar{K} K$ threshold. While 

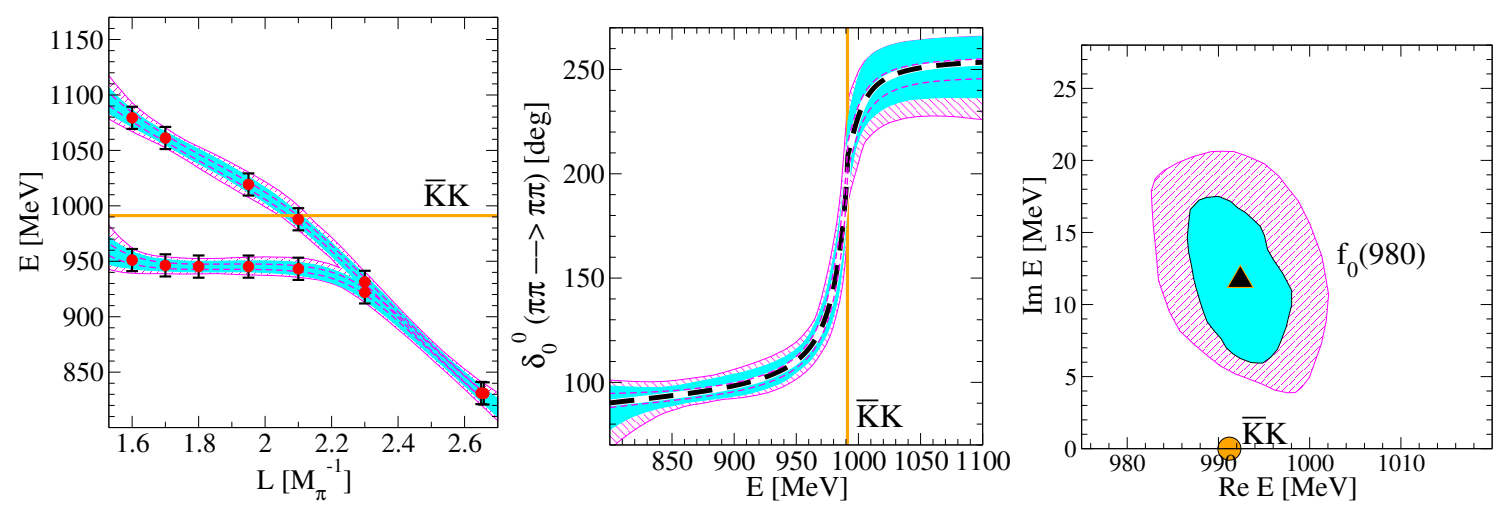

Figure 1: Left: generated synthetic lattice data for the energy region of the $f_{0}(980)$. Shown are two sets. The one with the visible plateau is evaluated with periodic boundary condition, the other one with twisted boundary conditions of $\vec{\theta}=(\pi, \pi, \pi)$. Center: Extracted phase shift close to the $K \bar{K}$ threshold. Right: complex energy plane. The reconstructed $f_{0}(980)$ pole position (colored areas) matches with the exact value (triangle). See text for further explanations.

for periodic boundary conditions, $\tilde{G}$ has a pole at threshold $(\vec{q}=(0,0,0))$, this is obviously not any more the case for $\vec{\theta} \neq(0,0,0)$.

Using the Chiral Unitary Approach of Ref. [55], one can generate realistic lattice eigenvalues for different box sizes $L$ as shown in Fig. 1 to the left. The data $E_{n}(L)$ are fitted by using linear functions in the potential $V_{i j}$,

$$
V_{i j}(s)=a_{i j}+b_{i j}\left(s-4 M_{K}^{2}\right)
$$

where $i, j$ are indices for the $\pi \pi$ and the $K \bar{K}$ channels with $a_{i j}=a_{j i}$ and $b_{i j}=b_{j i}$ for $i \neq j$. As mentioned before, the $V$ are indeed smooth functions of $s$ by construction. With the $a, b$ determined, the original scattering equation (2.1) can be solved. The extracted infinite-volume phase shift is shown in the middle panel of Fig. 1, as a function of $E=\sqrt{s}$. To the right in Fig. 1 the complex plane of scattering energy is shown with the reconstructed pole position of the $f_{0}(980)$ (shaded areas). As the data had been originally generated from the approach of Ref. [55], the exact pole position is known (triangle in the figure). The figure shows thus, that it is indeed possible to reliably reconstruct the resonance.

The shaded areas in Fig. 1 indicate how the given uncertainties in the eigenvalues propagate to the extracted phase shift and pole position. The pink hatched area, in contrast to the cyan one, takes also into account that the centroids of the generated pseudo data scatter according to the assumed uncertainty (size of the error bars).

This example shows that making minimal assumptions on the energy dependence of the kernel, one can relate lattice eigenvalues at different $L$ and with different boundary conditions to extract the amplitude. Also, the use of Chiral Unitary amplitudes to predict eigenvalues - possibly in combination with a chiral extrapolation - allows to choose appropriate lattice setups, because it can be estimated which lattice configuration is most effective to study a given resonance, and which precision is needed to do so.

Another question is whether partial twisting of the valence quarks, that is more economical in terms of computational requirements, is equivalent to full twisting. This question has been 

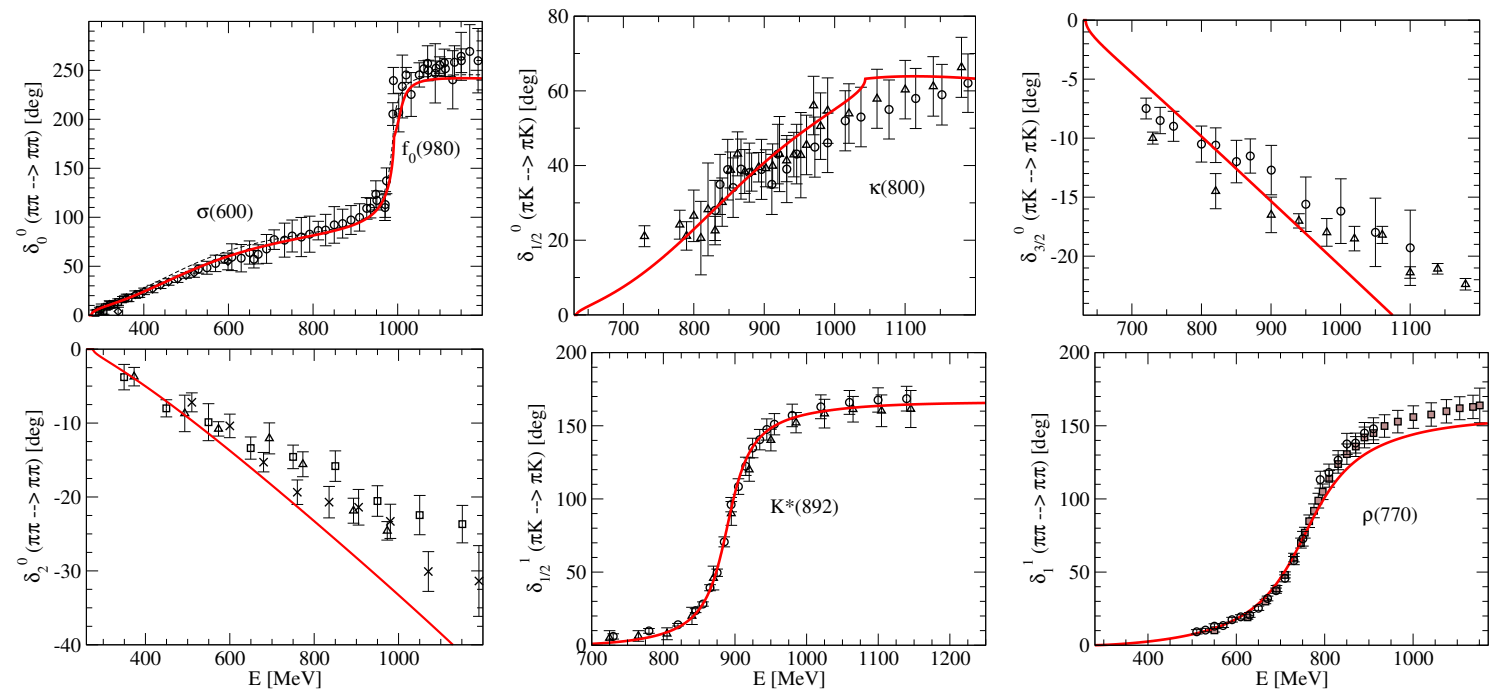

Figure 2: Fit of the Low Energy Constants of the amplitude of Ref. [61] to phase shift data, performed in Ref. [60]. For data reference, see Ref. [60].

addressed in the past $[56,57]$ and has been addressed on this conference by A. Rusetsky in a new study. Using the non-relativistic EFT technique in a finite volume, the authors of Ref. [58] have derived the Lüscher equation for partially twisted boundary conditions (adapting methods of partially quenched CHPT [59]), for coupled-channel $\pi \eta-K \bar{K}$ scattering. The final result is remarkably simple: if in the channel with $I=I_{3}=1$ the light quarks are subject to twisting, the partially twisted Lüscher equation is equivalent to the fully twisted one, despite the presence of annihilation diagrams. If, on the contrary, partial twisting of the strange quark is performed, the physically interesting part of the spectrum is not affected. Other scenarios are also possible and can be investigated by using the same methods.

\subsection{Using CHPT to stabilize the resonance extraction}

While the $a, b$ in Eq. (2.1) are free real fit parameters, one can use Chiral Perturbation Theory to stabilize the resonance extraction, as demonstrated in Ref. [60]. For this, the amplitude of Ref. [61] has been adopted and the Low Energy Constants were fitted to available phase shift data as shown in Fig. 2. Evaluating the same approach in the finite volume, the level spectrum shown in Fig. 3 was predicted. As in the previous example, synthetic data were generated, but in this case they were fitted with the ansatz

$$
V^{\mathrm{fit}}=\left(\frac{V_{2}-V_{4}^{\mathrm{fit}}}{V_{2}^{2}}\right)^{-1}, \quad V_{4}^{\mathrm{fit}}=a+b\left(s-s_{0}\right)+c\left(s-s_{0}\right)^{2}+d\left(s-s_{0}\right)^{3}+\cdots
$$

that has been written for the one-channel case for simplicity. Here, $V_{2} \equiv V_{\mathrm{LO}}$ is the fixed lowest order (LO) contribution of the chiral expansion. In other words, the kernel is parameterized similarly as in the inverse amplitude method [61], leaving the LO terms $V_{2}$ as it is, and expand $V_{4}$ in powers of $s$. As shown in Ref. [60], including the LO terms considerably helps stabilize the phase shift and resonance pole extraction. It would be also possible to include the next-to-leading order 

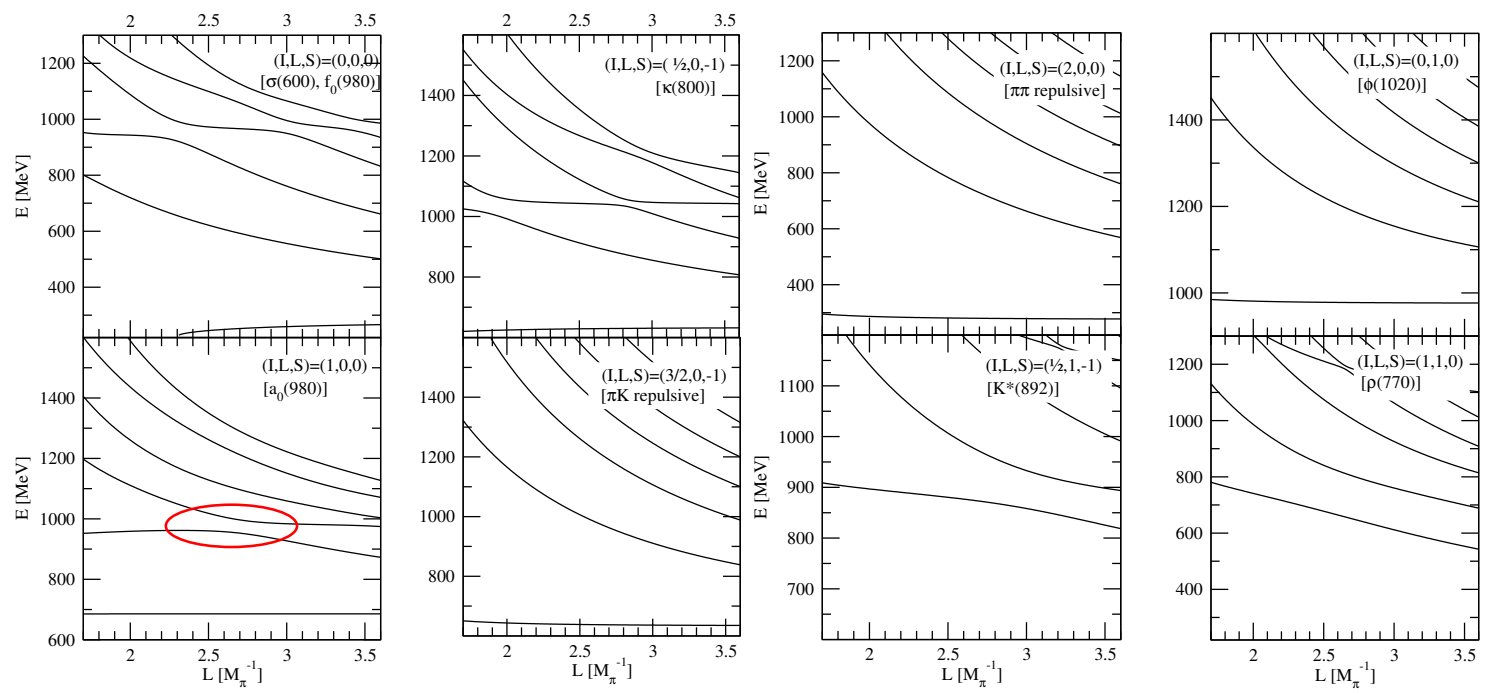

Figure 3: Level spectrum predicted from the approach of Ref. [60].

contribution explicitly, plus a polynomial of higher order, and fit low-energy constants and polynomial coefficients together. It is clear that when proceeding along the ideas outlined here, one does not have to evaluate phase shifts to get the resonance parameters, but can directly evaluate the amplitude on the second Riemann sheet and determine pole position and residues.

On the Lattice2013 conference, new results on the $a_{0}(980)$ resonance have been presented [62] by the ETMC group (see also Refs. [63, 64]). Although operators of the tetraquark state are introduced, only two states are observed that are rather of the two-particle type $\left(K \bar{K}, \eta_{s} \pi\right)$. Note that at the physical point, the chiral unitary calculation as well predicts only two scattering states as highlighted in Fig 3 (though there is a resonance pole of the $a_{0}(980)$ ). Further studies in the two-channel framework could shed light on the issue.

\subsection{Moving frames: partial wave mixing and coupled channels}

The Lüscher formalism [38, 39] was derived for a pair of particles with total zero-momentum. The generalization to a moving frame has been done in [56, 65-75] and in Ref. [76] for the general case of different spins. In Ref. [77], the formalism for the Chiral Unitary framework has been developed. On the Lattice 2013 conference, several examples were presented that show the power of the method: for the $\rho$ (770) quantum numbers (HadronSpectrum Collaboration [44]), and for the repulsive $\pi K$ phase shift by D. Wilson. For earlier work of the group, see e.g. the simultaneous extraction of repulsive $S$ - and $D$-waves in $\pi \pi$ scattering to unprecedented precision [71, 78].

In Ref. [77], the concepts of moving frames and coupled-channel scattering were brought together. This is illustrated for the example of the $S$ - and $D$-wave system in isospin zero mesonmeson scattering. For the $S$-wave, the channels $\pi \pi$ and $K \bar{K}$ are allowed, while for simplicity only the $\pi \pi$ channel is included for the $D$-wave. In multi-channel scattering, the scattering kernel $V$ and the finite-volume propagator $\tilde{G}$ can be introduced as matrices. When partial wave mixing is considered in addition, the matrices are simply given by the product of the channel space and the space of partial waves. As an example, for the boosts $\vec{P}=(2 \pi / L)(0,0,1),(2 \pi / L)(1,1,1)$, and 
$(2 \pi / L)(0,0,2)$ (representation $\left.A_{1}^{+}\right)$one has

$$
V=\left(\begin{array}{ccc}
V_{S}^{(11)} & V_{S}^{(12)} & 0 \\
V_{S}^{(21)} & V_{S}^{(22)} & 0 \\
0 & 0 & V_{D}^{(22)}
\end{array}\right), \quad \tilde{G}=\left(\begin{array}{ccc}
\tilde{G}_{00,00}^{R(1)} & 0 & 0 \\
0 & \tilde{G}_{00,00}^{R(2)} & \tilde{G}_{00,20}^{R(2)} \\
0 & \tilde{G}_{20,00}^{R(2)} & \tilde{G}_{20,20}^{R(2)}
\end{array}\right)
$$

where the lower index in $V$ indicates the partial wave and the upper index the channels (1: $K \bar{K}, 2$ : $\pi \pi$ ). The lower indices for $G$ indicate the $\ell m, \ell^{\prime} m^{\prime}$. The partial wave mixing occurs through the off-diagonal elements of $\tilde{G}$, while the channel mixing is provided by the off-diagonal elements of $V$.

In Fig. 4 to the top, the predicted levels for different boosts are shown. Moving frames provide the additional advantage, that the levels cover the resonance region of the $\sigma(600)$. The lower panel to the left shows different fits to synthetic data generated from these eigenvalues. The power, up to which the ansatz of Eq. (2.1) is expanded, is indicated in the figure. The area indicates the expected uncertainty if terms up to $s^{3}$ are allowed in the expansion. Expansion to even higher orders do not change the extracted pole position any more, but only increase the uncertainties, which provides a criterion up to which order the expansion should be carried out. For more details, see Ref. [77]. To the right in Fig. 4, a comparable fit is shown, but refraining from moving frames. In that case, one needs eigenvalues at six or more different box sizes to obtain a comparable accuracy.

Relating different energies by an expansion of the type of Eq. (2.1) is particularly suited for the moving frame case, because to disentangle partial waves, one needs the eigenvalues of different representations at the same energy. This is, like in the multi-channel case, difficult to achieve. With minimal assumptions on the smoothness of the amplitudes, one can overcome this problem.

\section{Baryon resonances: $N(1535) 1 / 2^{-}, N(1650) 1 / 2^{-}$, and $\Lambda(1405) 0^{-}$}

Recently, first results on the spectrum of excited baryons including the decay dynamics have appeared [79]. These results by the Graz group have been presented at the Lattice 2013 conference [79]. A few phase shift points could be extracted using Lüscher's method, and first evidence for the $N(1535) 1 / 2^{-}$and the $N(1650) 1 / 2^{-}$resonances could be found.

Results from Ref. [80] show that when quark masses drop further, an unexpected behavior for the chiral extrapolation could occur. For this, the next-to-leading order chiral unitary calculation of Ref. [81] (see also Ref. [82]) was extrapolated to different lattice setups. In Fig. 3 to the left, the fit of the low-energy constants to the partial wave data of Ref. [52] is shown. The lower panel to the left shows different Riemann sheets connected to different parts of the physical axis. Poles of the $N(1535) 1 / 2^{-}$and the $N(1650) 1 / 2^{-}$are found, as well as a shadow pole $N^{\prime}$. As quark masses rise from the physical point, thresholds start moving as well. For the QCDSF setup of Ref. [83], the chiral extrapolation leads to the amplitude shown to the upper right. In particular, thresholds move faster that the resonance poles in this case. The prominent resonance structure visible in the amplitude is not induced by any of the original $N(1535) 1 / 2^{-}$and the $N(1650) 1 / 2^{-}$ resonance poles. Rather, it results from a shadow pole that is invisible at physical quark masses and becomes uncovered at unphysical quark masses. In conclusion, coupled channel dynamics induces here a complication of a different type than those discussed before. In Ref. [80], the finite-volume 

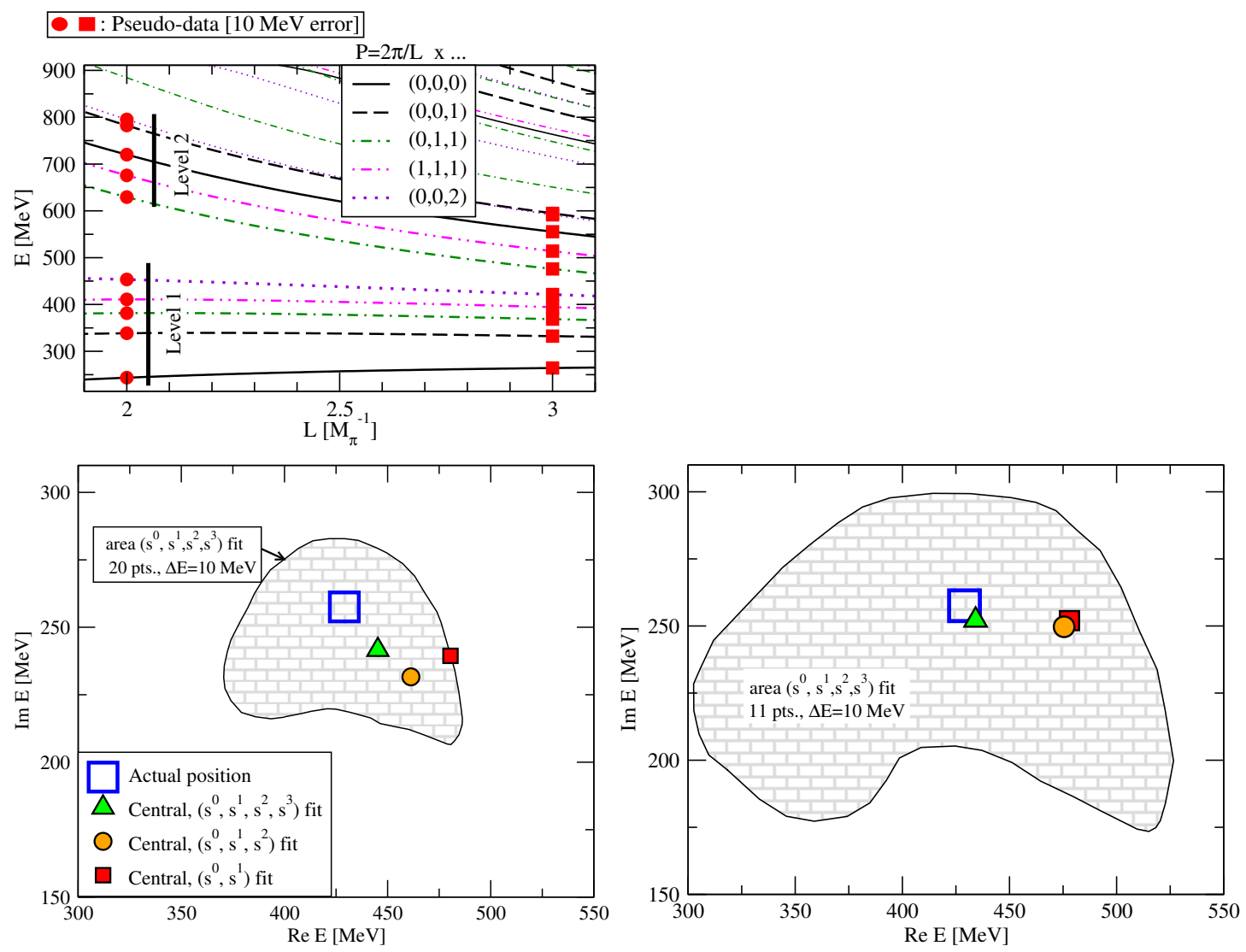

Figure 4: Upper panel: Prediction of the levels in the scalar-isoscalar sector for different boosts. The lower panel to the left shows the extracted $\sigma(600)$ pole positions, from data at two different box sizes. For comparison, the right panel shows the extracted pole positions without moving frames but using 6 different box sizes. For further explanations, see text.
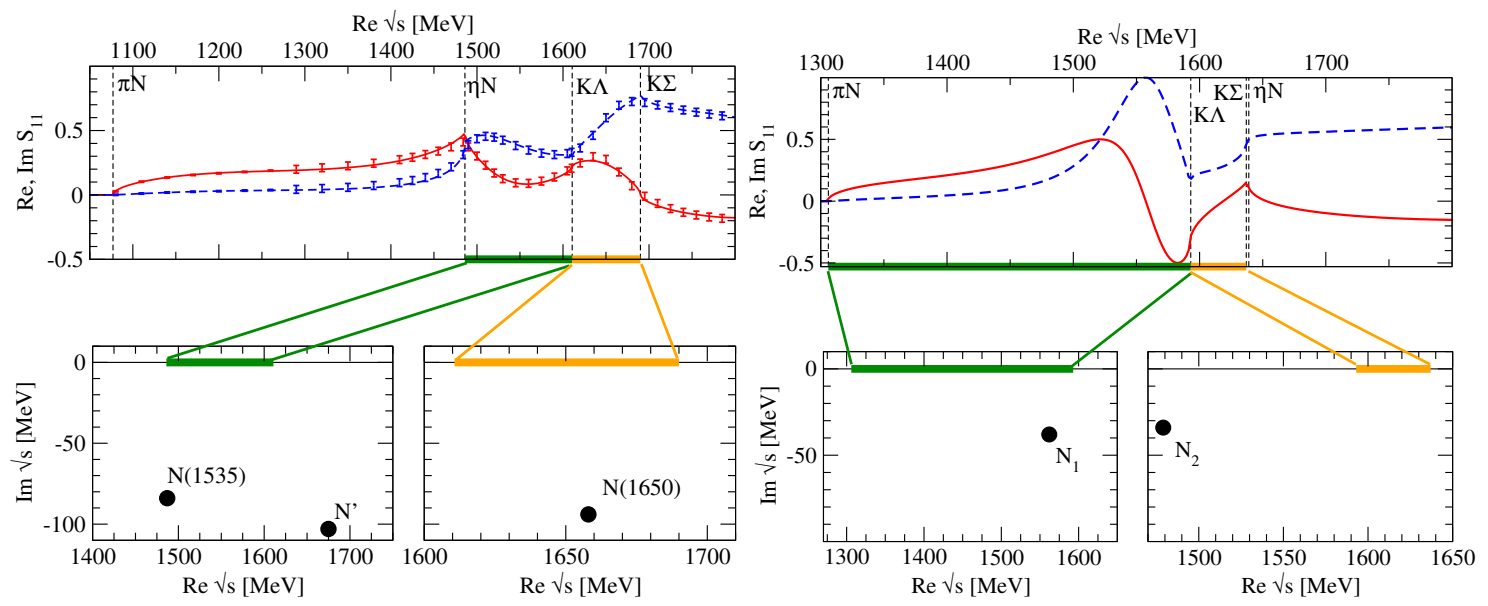

Figure 5: Results from Ref. [80]. Left: Fit of low-energy constants to the $\pi N$ partial wave data of Ref. [52] (shown are the real (red) and the imaginary parts (blue) of the amplitude). The lower plots show pole positions. Right: Chiral extrapolation to a QCDSF lattice setup [83]. The $\pi N, \eta N, K \Lambda$, and $K \Sigma$ thresholds are indicated in the figures. See text for further explanations. 
spectrum is predicted as well, and different strategies to extract the resonances are discussed, in particular using twisted boundary conditions.

Pioneering work for the coupled-channel $\bar{K} N, \pi \Sigma$ system and the $\Lambda(1405)$ in the finite volume has been carried out in Ref. [49]; the lattice levels for the $\Lambda(1405)$ quantum numbers were predicted in Ref. [84] using a dynamical coupled channel model in the finite volume; strategies to determine the two $\Lambda(1405)$ states from lattice results were discussed in Ref. [85], using moving frames. First results of actual lattice calculation by the Adelaide group have appeared recently and were presented on the Lattice 2013 conference [23] (see also Ref. [86]).

\section{Outlook}

As quark masses drop, resonance can decay in the finite volume. Lüscher's method provides the model-independent method to extrapolate amplitudes to the infinite volume. As scattering problems become more complex and new decay channels open, the multi-channel extension of the method will become relevant. The formalism can be combined with moving frames and modified boundary conditions, to obtain more lattice eigenvalues, to disentangle partial waves, or to disentangle resonances from threshold effects. To improve the accuracy of the result, interpolations in energy, with minimal assumptions on the smoothness of the amplitude, allow to relate eigenvalues at different energies to each other. Such fits can be stabilized by Chiral Parturbation Theory.

Three-body scattering problems in the finite volume start to become of high interest, for example triggered by recent JLab calculations of excited mesons, partly with exotic quantum numbers [26], that decay to multi-meson final states. See also Ref. [27] for a first calculation of the $a_{1}$ meson decaying into $\pi \rho$. A generalized scheme for three-body systems has been presented on this conference by M. Hansen and S. Sharpe. Other developments in this direction are given in Refs. [74, 87-90]. With the rapid conceptual progress of methods, it will be possible to address the consequences for the finite-volume spectrum of the Roper resonance, coming from its large $\pi \pi N$ inelasticties.

\section{Acknowledgments}

The author thanks Raúl Briceño, G. Engel, M. Hansen, C. Lang, B. Menadue, M. Petschlies, A. Rusetsky, G. Schierholz, S. Sharpe, C. Thomas, C. Urbach, M. Wagner, D. Wilson for interesting discussions and transparencies.

\section{References}

[1] Y. Nakahara, M. Asakawa and T. Hatsuda, Phys. Rev. D 60 (1999) 091503. [hep-lat/9905034].

[2] K. Sasaki, S. Sasaki and T. Hatsuda, Phys. Lett. B 623 (2005) 208. [hep-lat/0504020].

[3] N. Mathur, A. Alexandru, Y. Chen, S. J. Dong, T. Draper, I. Horvath, F. X. Lee and K. F. Liu et al., Phys. Rev. D 76 (2007) 114505. [hep-ph/0607110].

[4] S. Basak, R. G. Edwards, G. T. Fleming, K. J. Juge, A. Lichtl, C. Morningstar, D. G. Richards and I. Sato et al., Phys. Rev. D 76 (2007) 074504. [arXiv:0709.0008 [hep-lat]]. 
[5] J. Bulava, R. G. Edwards, E. Engelson, B. Joo, H-W. Lin, C. Morningstar, D. G. Richards and S. J. Wallace, Phys. Rev. D 82 (2010) 014507. [arXiv:1004.5072 [hep-lat]].

[6] C. Morningstar, A. Bell, J. Bulava, E. Engelson, J. Foley, K. J. Juge, D. Lenkner and M. Peardon et al., AIP Conf. Proc. 1257 (2010) 779. [arXiv:1002.0818 [hep-lat]].

[7] J. Foley, J. Bulava, K. J. Juge, C. Morningstar, M. Peardon and C. H. Wong, AIP Conf. Proc. 1257 (2010) 789. [arXiv:1003.2154 [hep-lat]].

[8] M. G. Alford and R. L. Jaffe, Nucl. Phys. B 578 (2000) 367. [hep-lat/0001023].

[9] T. Kunihiro et al. [SCALAR Collaboration], Phys. Rev. D 70 (2004) 034504. [hep$\mathrm{ph} / 0310312]$.

[10] F. Okiharu, T. Doi, H. Ichie, H. Iida, N. Ishii, M. Oka, H. Suganuma and T. T. Takahashi, hep-ph/0507187.

[11] H. Suganuma, K. Tsumura, N. Ishii and F. Okiharu, PoS LAT 2005 (2006) 070. [heplat/0509121].

[12] H. Suganuma, K. Tsumura, N. Ishii and F. Okiharu, Prog. Theor. Phys. Suppl. 168 (2007) 168. [arXiv:0707.3309 [hep-lat]].

[13] C. McNeile and C. Michael [UKQCD Collaboration], Phys. Rev. D 74 (2006) 014508. [arXiv:hep-lat/0604009];

[14] A. Hart et al. [UKQCD Collaboration], Phys. Rev. D 74 (2006) 114504. [hep-lat/0608026].

[15] H. Wada, T. Kunihiro, S. Muroya, A. Nakamura, C. Nonaka and M. Sekiguchi, Phys. Lett. B 652 (2007) 250. [arXiv:hep-lat/0702023].

[16] S. Prelovsek, C. Dawson, T. Izubuchi, K. Orginos and A. Soni, Phys. Rev. D 70 (2004) 094503. [hep-lat/0407037].

[17] S. Prelovsek, T. Draper, C. B. Lang, M. Limmer, K. F. Liu, N. Mathur and D. Mohler, Conf. Proc. C 0908171 (2009) 508. [arXiv:1002.0193 [hep-ph]].

[18] S. Prelovsek, T. Draper, C. B. Lang, M. Limmer, K.-F. Liu, N. Mathur and D. Mohler, Phys. Rev. D 82 (2010) 094507. [arXiv:1005.0948 [hep-lat]].

[19] H. -W. Lin et al. [Hadron Spectrum Collaboration], Phys. Rev. D 79 (2009) 034502. [arXiv:0810.3588 [hep-lat]].

[20] C. Gattringer, C. Hagen, C. B. Lang, M. Limmer, D. Mohler and A. Schäfer, Phys. Rev. D 79 (2009) 054501. [arXiv:0812.1681 [hep-lat]].

[21] G. P. Engel et al. [BGR [Bern-Graz-Regensburg] Collaboration], Phys. Rev. D 82 (2010) 034505. [arXiv:1005.1748 [hep-lat]]. 
[22] M. S. Mahbub, W. Kamleh, D. B. Leinweber, A. O Cais and A. G. Williams, Phys. Lett. B 693 (2010) 351. [arXiv:1007.4871 [hep-lat]].

[23] B. J. Menadue, W. Kamleh, D. B. Leinweber and M. S. Mahbub, Phys. Rev. Lett. 108 (2012) 112001. [arXiv:1109.6716 [hep-lat]].

[24] R. G. Edwards, J. J. Dudek, D. G. Richards and S. J. Wallace, Phys. Rev. D 84 (2011) 074508 [arXiv:1104.5152 [hep-ph]].

[25] J. J. Dudek, R. G. Edwards, B. Joo, M. J. Peardon, D. G. Richards and C. E. Thomas, Phys. Rev. D 83, 111502 (2011) [arXiv:1102.4299 [hep-lat]].

[26] J. J. Dudek, R. G. Edwards, M. J. Peardon, D. G. Richards and C. E. Thomas, Phys. Rev. D 82, 034508 (2010) [arXiv:1004.4930 [hep-ph]].

[27] S. Prelovsek, C. B. Lang, D. Mohler and M. Vidmar, PoS LATTICE 2011 (2011) 137. [arXiv:1111.0409 [hep-lat]].

[28] Z. Fu, JHEP 1201, 017 (2012).

[29] C. B. Lang, L. Leskovec, D. Mohler and S. Prelovsek, arXiv:1207.3204 [hep-lat].

[30] J. J. Dudek, R. G. Edwards, P. Guo and C. E. Thomas, Phys. Rev. D 88, 094505 (2013) [Phys. Rev. D 88, 094505 (2013)] [arXiv:1309.2608 [hep-lat]].

[31] G. P. Engel, C. B. Lang, M. Limmer, D. Mohler and A. Schäfer, Phys. Rev. D 85, 034508 (2012) [arXiv:1112.1601 [hep-lat]].

[32] G. P. Engel, C. B. Lang, D. Mohler and A. Schafer, Phys. Rev. D 87, 074504 (2013) [arXiv:1301.4318 [hep-lat]].

[33] V. Bernard, U.-G. Meißner and A. Rusetsky, Nucl. Phys. B 788 (2008) 1. [hep-lat/0702012 [HEP-LAT]].

[34] V. Bernard, M. Lage, U.-G. Meißner and A. Rusetsky, JHEP 0808 (2008) 024 [arXiv:0806.4495 [hep-lat]].

[35] V. Bernard, D. Hoja, U.-G. Meißner and A. Rusetsky, JHEP 0906 (2009) 061. [arXiv:0902.2346 [hep-lat]].

[36] V. Bernard, M. Lage, U.-G. Meißner and A. Rusetsky, JHEP 1101 (2011) 019 [arXiv:1010.6018 [hep-lat]].

[37] M. Döring, U.-G. Meißner, E. Oset and A. Rusetsky, Eur. Phys. J. A 47, 139 (2011) [arXiv:1107.3988 [hep-lat]].

[38] M. Lüscher, Commun. Math. Phys. 105 (1986) 153.

[39] M. Lüscher, Nucl. Phys. B 354 (1991) 531. 
[40] M. Göckeler et al. [QCDSF Collaboration], PoS LATTICE 2008, 136 (2008) [arXiv:0810.5337 [hep-lat]].

[41] C. B. Lang, D. Mohler, S. Prelovsek and M. Vidmar, Phys. Rev. D 84 (2011) 054503 [arXiv:1105.5636 [hep-lat]].

[42] C. S. Pelissier, A. Alexandru and F. X. Lee, PoS LATTICE 2011, 134 (2011) [arXiv:1111.2314 [hep-lat]].

[43] C. Pelissier and A. Alexandru, Phys. Rev. D 87, 014503 (2013) [arXiv:1211.0092 [hep-lat]].

[44] J. J. Dudek, R. G. Edwards and C. E. Thomas, Phys. Rev. D 87, no. 3, 034505 (2013) [arXiv:1212.0830 [hep-ph]].

[45] C. B. Lang, L. Leskovec, D. Mohler and S. Prelovsek, arXiv:1310.4958 [hep-lat].

[46] S. Prelovsek, L. Leskovec, C. B. Lang and D. Mohler, Phys. Rev. D 88 (2013) 054508 [arXiv:1307.0736 [hep-lat]].

[47] S. He, X. Feng and C. Liu, JHEP 0507 (2005) 011.

[48] C. Liu, X. Feng and S. He, Int. J. Mod. Phys. A 21 (2006) 847.

[49] M. Lage, U.-G. Meißner and A. Rusetsky, Phys. Lett. B 681 (2009) 439. [arXiv:0905.0069 [hep-lat]].

[50] D. Jido, J. A. Oller, E. Oset, A. Ramos and U. G. Meissner, Nucl. Phys. A 725, 181 (2003) [nucl-th/0303062].

[51] C. Alexandrou, J. W. Negele, M. Petschlies, A. Strelchenko and A. Tsapalis, Phys. Rev. D 88, 031501 (2013) [arXiv:1305.6081 [hep-lat]].

[52] R. L. Workman, M. W. Paris, W. J. Briscoe and I. I. Strakovsky, Phys. Rev. C 86, 015202 (2012) [arXiv:1202.0845 [hep-ph]].

[53] S. Ceci, M. Döring, C. Hanhart, S. Krewald, U.-G. Meißner and A. Švarc, Phys. Rev. C 84, 015205 (2011) [arXiv:1104.3490 [nucl-th]].

[54] M. Albaladejo, J. A. Oller, E. Oset, G. Rios and L. Roca, JHEP 1208 (2012) 071 [arXiv:1205.3582 [hep-lat]].

[55] J. A. Oller, E. Oset, Chiral symmetry amplitudes in the $S$ wave isoscalar and isovector channels and the $\sigma, f_{0}(980), a_{0}(980)$ scalar mesons, Nucl. Phys. A620, 438 (1997) [arXiv:hep$\mathrm{ph} / 9702314]$.

[56] C. h. Kim, C. T. Sachrajda and S. R. Sharpe, Nucl. Phys. B 727 (2005) 218.

[57] C. T. Sachrajda and G. Villadoro, Phys. Lett. B 609, 73 (2005) [hep-lat/0411033].

[58] D. Agadjanov, U.-G. Meißner and A. Rusetsky, arXiv:1310.7183 [hep-lat]. 
[59] S. R. Sharpe and N. Shoresh, Phys. Rev. D 64, 114510 (2001) [hep-lat/0108003].

[60] M. Döring and U.-G. Meißner, JHEP 1201 (2012) 009 [arXiv:1111.0616 [hep-lat]].

[61] J. A. Oller, E. Oset, J. R. Peláez, Meson meson interaction in a nonperturbative chiral approach, Phys. Rev. D59, 074001 (1999); [Erratum-ibid. D 60, 099906 (1999)]; [Erratum-ibid. D 75, 099903 (2007)]; [arXiv:hep-ph/9804209].

[62] M. Wagner, A. Abdel-Rehim, C. Alexandrou, M. Gravina, G. Koutsou, M. Dalla Brida, L. Scorzato and C. Urbach, arXiv:1309.0850 [hep-lat].

[63] M. Wagner, C. Alexandrou, M. Gravina, J. O. Daldrop, C. Urbach, M. Dalla Brida, L. Scorzato and C. Wiese, PoS ConfinementX , 108 (2012) [arXiv:1212.1648 [hep-lat]].

[64] C. Alexandrou, J. O. Daldrop, M. Dalla Brida, M. Gravina, L. Scorzato, C. Urbach and M. Wagner, JHEP 1304, 137 (2013) [arXiv:1212.1418].

[65] K. Rummukainen and S. A. Gottlieb, Nucl. Phys. B 450 (1995) 397 [hep-lat/9503028].

[66] S. Bour, S. König, D. Lee, H.-W. Hammer and U.-G. Meißner, Phys. Rev. D 84 (2011) 091503.

[67] S. R. Beane et al. [NPLQCD Collaboration], Phys. Rev. D 85 (2012) 034505.

[68] Z. Davoudi and M. J. Savage, Phys. Rev. D 84 (2011) 114502.

[69] Z. Fu, Phys. Rev. D 85 (2012) 014506.

[70] L. Leskovec and S. Prelovsek, arXiv:1202.2145 [hep-lat].

[71] J. J. Dudek, R. G. Edwards and C. E. Thomas, Phys. Rev. D 86, 034031 (2012) [arXiv:1203.6041 [hep-ph]].

[72] M. T. Hansen and S. R. Sharpe, Phys. Rev. D 86, 016007 (2012) [arXiv:1204.0826 [hep-lat]].

[73] R. A. Briceño and Z. Davoudi, arXiv:1204.1110 [hep-lat].

[74] S. Kreuzer and H. W. Grießhammer, Eur. Phys. J. A 48 (2012) 93. [arXiv:1205.0277 [nuclth]].

[75] R. A. Briceño, Z. Davoudi, T. Luu and M. J. Savage, Phys. Rev. D 88, 114507 (2013) [arXiv:1309.3556 [hep-lat]].

[76] M. Göckeler, R. Horsley, M. Lage, U.-G. Meißner, P. E. L. Rakow, A. Rusetsky, G. Schierholz and J. M. Zanotti, Phys. Rev. D 86 (2012) 094513 [arXiv:1206.4141 [hep-lat]].

[77] M. Döring, U.-G. Meißner, E. Oset and A. Rusetsky, Eur. Phys. J. A 48 (2012) 114 [arXiv:1205.4838 [hep-lat]].

[78] J. J. Dudek, R. G. Edwards, M. J. Peardon, D. G. Richards and C. E. Thomas, Phys. Rev. D 83, 071504 (2011) [arXiv:1011.6352 [hep-ph]]. 
[79] C. B. Lang and V. Verduci, Phys. Rev. D 87 (2013) 5, 054502 [arXiv:1212.5055].

[80] M. Döring, M. Mai and U.-G. Meißner, Phys. Lett. B 722 (2013) 185 [arXiv:1302.4065 [heplat]].

[81] P. C. Bruns, M. Mai and U.-G. Meißner, Phys. Lett. B 697, 254 (2011) [arXiv:1012.2233 [nucl-th]].

[82] M. Mai, P. C. Bruns and U.-G. Meißner, Phys. Rev. D 86, 094033 (2012) [arXiv:1207.4923 [nucl-th]].

[83] W. Bietenholz, V. Bornyakov, M. Gockeler, R. Horsley, W. G. Lockhart, Y. Nakamura, H. Perlt and D. Pleiter et al., Phys. Rev. D 84, 054509 (2011) [arXiv:1102.5300 [hep-lat]].

[84] M. Döring, J. Haidenbauer, U.-G. Meißner and A. Rusetsky, Eur. Phys. J. A 47 (2011) 163 [arXiv:1108.0676 [hep-lat]].

[85] A. Martinez Torres, M. Bayar, D. Jido and E. Oset, arXiv:1202.4297 [hep-lat].

[86] J. M. M. Hall, A. C. -P. Hsu, D. B. Leinweber, A. W. Thomas and R. D. Young, Phys. Rev. D 87, 094510 (2013) [arXiv:1303.4157 [hep-lat]].

[87] K. Polejaeva and A. Rusetsky, Eur. Phys. J. A 48, 67 (2012) [arXiv:1203.1241 [hep-lat]].

[88] L. Roca and E. Oset, Phys. Rev. D 85 (2012) 054507.

[89] R. A. Briceno and Z. Davoudi, Phys. Rev. D 87 (2013) 094507 [arXiv:1212.3398 [hep-lat]].

[90] P. Guo, J. Dudek, R. Edwards and A. P. Szczepaniak, Phys. Rev. D 88, 014501 (2013) [arXiv:1211.0929 [hep-lat]]. 